\title{
A global phosphoproteomics analysis of adult Fasciola gigantica by LC-MS/MS
}

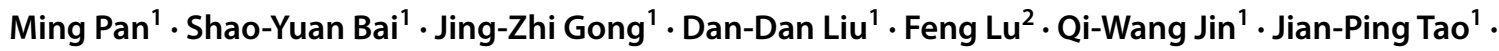 \\ Si-Yang Huang ${ }^{1}$ (i)
}

Received: 17 October 2021 / Accepted: 29 December 2021 / Published online: 5 January 2022

(c) The Author(s), under exclusive licence to Springer-Verlag GmbH Germany, part of Springer Nature 2022

\begin{abstract}
Protein phosphorylation plays key roles in a variety of essential cellular processes. Fasciola gigantica is a tropical liver fluke causing hepatobiliary disease fascioliasis, leading to human health threats and heavy economic losses. Although the genome and protein kinases of $F$. gigantica provided new insights to understand the molecular biology and etiology of this parasite, there is scant knowledge of protein phosphorylation events in F. gigantica. In this study, we characterized the global phosphoproteomics of adult $F$. gigantica by phosphopeptide enrichment-based LC-MS/MS, a high-throughput analysis to maximize the detection of a large repertoire of phosphoproteins and phosphosites. A total of 1030 phosphopeptides with 1244 phosphosites representing $635 \mathrm{~F}$. gigantica phosphoproteins were identified. The phosphoproteins were involved in a wide variety of biological processes including cellular, metabolic, and single-organism processes. Meanwhile, these proteins were found predominantly in cellular components like membranes and organelles with molecular functions of binding (51.3\%) and catalytic activity (40.6\%). The KEGG annotation inferred that the most enriched pathways of the phosphoproteins included tight junction, spliceosome, and RNA transport (each one contains 15 identified proteins). Combining the reports in other protozoa and helminths, the phosphoproteins identified in this work play roles in metabolic regulation and signal transduction. To our knowledge, this work performed the first global phosphoproteomics analysis of adult $F$. gigantica, which provides valuable information for development of intervention strategies for fascioliasis.
\end{abstract}

Keywords Fasciola gigantica $\cdot$ Mass spectrometry $\cdot$ Phosphoproteomics $\cdot$ Phosphoprotein · Annotation

\section{Background}

Fasciola spp. are parasitic food-borne trematodes causing hepatobiliary disease-fascioliasis, leading to extensive liver tissue damage and cirrhosis (Good and Scherbak 2021).

Section Editor: Xing-Quan ZHU

Ming Pan and Shao-Yuan Bai contributed equally to this work.

Si-Yang Huang

siyang.huang@hotmail.com

1 Institute of Comparative Medicine, College of Veterinary

Medicine, Yangzhou University, and Jiangsu CoInnovation Center for Prevention and Control of Important Animal Infectious Diseases and Zoonosis, and Jiangsu Key Laboratory of Zoonosis, Yangzhou, Jiangsu Province 225009, People's Republic of China

2 School of Medicine, Yangzhou University, Yangzhou 225009, People's Republic of China
Fascioliasis is endemic worldwide infecting 17 million people from 61 countries, and 180 million people are at risk of infection (Mas-Coma et al. 2019; McManus 2020). Meanwhile, these trematodes infect approximately 600 million domestic ruminants with a significantly negative effect on their live weight gains, causing profound damage to animal productivity and economic losses in excess of US $\$ 3$ billion per year (Cwiklinski et al. 2016; Hayward et al. 2021). As the only drug effective against the infection, triclabendazole (TCBZ) was recently approved to be used by the FDA, and there is currently no therapeutic alternative to treat human fascioliasis. However, someone treated with TCBZ still continue excreting eggs through feces despite a standard care regimen (Marcos et al. 2021). Additionally, emerging hybridization and introgression between Fasciola gigantica and Fasciola hepatica make the therapy and control of fascioliasis more difficult (Calvani and Slapeta 2021).

Post-translational modifications (PTMs) are biochemical changes to regulate the modified proteins, which are 
indispensable for their functional diversities (Mann and Jensen 2003). Therein, as one of the most important PTMs, reversible phosphorylation at serine, threonine, and tyrosine residues plays key roles in a variety of cellular processes including signal transduction, membrane transport, and metabolic regulation (Humphrey et al. 2015; Day et al. 2016). Protein kinases and protein phosphatases are involved to catalyze the protein phosphorylation/dephosphorylation processes via addition and removal of phosphoryl groups to substrates (Wang et al. 2020; Hirst et al. 2020). Particularly, protein kinases from Schistosoma and Leishmania as well as Toxoplasma had been identified as potential drug targets against these zoonotic parasites (Gaji et al. 2021; Wu et al. 2021; Efstathiou and Smirlis 2021). For $F$. gigantica, the draft genome (size: $1.04-1.13 \mathrm{~Gb}$ ) was assembled with a high degree of genomic polymorphism. Using DNA library construction and sequencing as well as bioinformatics analysis, a total of 20,858 genes were predicted, and 455 protein kinases were identified in 2020 (Pandey et al. 2020; Das et al. 2020). However, due to the limitations in genome annotation and functional study of these kinases, there is scant knowledge of protein phosphorylation events in $F$. gigantica. Recent progress has been made in the global phosphoproteomics analyses of various parasites (Hirst et al. 2020; Wang et al. 2020; Liu et al. 2020b), which made contributions to understanding the complex biology of these organisms and developing new anti-parasitic candidates.

To identify a large repertoire of phosphoproteins or establish a phosphoproteomics database, the high-throughput analysis of liquid chromatography-tandem mass spectrometry (LC-MS/MS) is currently the most common and powerful analytical platform for analyzing the phosphorylation state of parasites (Wang et al. 2019a; Broncel and Treeck 2020). In the present study, the phosphopeptide enrichmentbased LC-MS/MS was used to define the first global phosphoproteomics analysis of adult $F$. gigantica. Combining with functional annotations and biological connections, this work provides an overview and discusses current knowledge on phosphorylation of $F$. gigantica and enables us to develop new intervention strategies to control fascioliasis.

\section{Methods}

\section{Parasite preparation}

Adult Fasciola gigantica were isolated from the bile ducts of naturally infected buffaloes, washed thoroughly with phosphate-buffered saline (PBS, pH 7.4), and then incubated in RPMI 1640 medium at $37{ }^{\circ} \mathrm{C}$ for $1 \mathrm{~h}$. The flukes were frozen in liquid nitrogen and stored at $-80{ }^{\circ} \mathrm{C}$ until use.

\section{Protein extraction and digestion}

Protein extraction was conducted on three biological repeats from the frozen samples. Each sample was ground into powder and suspended in a lysis buffer ( $8 \mathrm{M}$ urea in $100 \mathrm{mM}$ triethyl ammonium bicarbonate, $\mathrm{pH} 8.5$ ) with $1 \%$ protease inhibitor cocktail (Merck, Denmark). Subsequently, the sample was ultrasonicated on ice at $30 \mathrm{~s}$ bursts for $10 \mathrm{~min}$. The lysate debris were eliminated by centrifugation at $12,000 \mathrm{~g}$ for $30 \mathrm{~min}$ at $4{ }^{\circ} \mathrm{C}$, and individual supernatants were collected and conducted to determine the protein concentration by a bicinchoninic acid (BCA) protein assay kit (Thermo Fisher Scientific, USA). Protein digestion was carried out as previously described (Liu et al. 2020b; Ren et al. 2018), with some modifications. Briefly, $300 \mu \mathrm{g}$ protein sample was reduced with $20 \mathrm{mM}$ dithiothreitol at $56^{\circ} \mathrm{C}$ for $30 \mathrm{~min}$, followed by alkylation with $100 \mathrm{mM}$ iodoacetamide at room temperature for $30 \mathrm{~min}$, then dissolved into urea solution with the addition of $25 \mathrm{mM} \mathrm{NH}_{4} \mathrm{HCO}_{3}$. Finally, the digestion process was conducted on a trypsin (Promega, USA) solution $\left(6 \mu \mathrm{g}\right.$ trypsin in $\left.40 \mu \mathrm{L} 100 \mathrm{mM} \mathrm{NH}_{4} \mathrm{HCO}_{3}\right)$ with a 1:100 ratio of trypsin-to-protein mass overnight at $37{ }^{\circ} \mathrm{C}$.

\section{Phosphopeptide enrichment}

After enzymatic hydrolysis, the peptide solution was lyophilized in vacuum and dissolved in a 2,5-dihydroxybenzoic acid (DHB) buffer (Thingholm et al. 2006). Phosphopeptides were enriched using a titanium dioxide $\left(\mathrm{TiO}_{2}\right)$ enrichment method (Wang et al. 2020; Larsen et al. 2007), with some modifications. Briefly, $\mathrm{TiO}_{2}$ beads (Thermo Fisher Scientific, USA) were mixed with peptides and incubated for $40 \mathrm{~min}$ at room temperature and then eluted with $1 \%$ ammonia solution and 30\% acetonitrile. Subsequently, phosphopeptides were freeze-dried and then dissolved with $20 \mu \mathrm{L}$ $0.1 \%$ formic acid (FA). Therein, $6 \mu \mathrm{L}$ sample was analyzed for LC-MS/MS analysis.

\section{LC-MS/MS}

LC-MS/MS analysis was performed using the Q Exactive Mass spectrometer (Thermo Fisher Scientific, USA) with a nanoliter flow rate HPLC liquid system-EASY-nLC1000 (Thermo Fisher Scientific, USA) according to the manufacturer's instructions.

The tryptic peptides were injected into the enrichment column EASY column SC001 traps $150 \mu \mathrm{m} * 20 \mathrm{~mm}$ RP-C18 (Thermo Fisher Scientific, USA) at an isocratic flow of $5 \mu \mathrm{L} /$ min of $2 \% \mathrm{CH}_{3} \mathrm{CN}$ containing $0.1 \% \mathrm{FA}$ (solvent A) for $6 \mathrm{~min}$ and then separated by the analytical column EASY column SC200 $150 \mu \mathrm{m} * 100 \mathrm{~mm}$ RP-C18 (Thermo Fisher Scientific, 
USA) at an isocratic flow of $400 \mathrm{~nL} / \mathrm{min}$. The solvent $\mathrm{A}$ and $84 \% \mathrm{CH}_{3} \mathrm{CN}$ in $0.1 \% \mathrm{FA}$ (solvent $\mathrm{B}$ ) were as the eluents. The gradient of solvent $\mathrm{B}$ was as follows: (i) $0-110 \mathrm{~min}, 0-55 \%$ solvent B; (ii) 110-118 min, 55-100\% B; and (iii) 118-120 min, maintaining at $100 \%$ B. Subsequently, the elution products were analyzed by the Q Exactive Mass spectrometer. The spectra of first-grade MS (MS1) were acquired during the scan scope of $300-1800 \mathrm{~m} / \mathrm{z}$ with a $2.0 \mathrm{kV}$ electrospray voltage, a resolution of 70,000, an automatic gain control (AGC) target of $3 \mathrm{e}^{6}$, and a maximum IT time of $50 \mathrm{~ms}$. The spectra of second-grade MS (MS2) were obtained using the following parameter: a resolution of 17,500 , a maximum injection time of $40 \mathrm{~ms}$, and an AGC target of $1 \mathrm{e}^{5}$. In addition, the mode of MS2 spectra was high-energy collisional dissociation (HCD), and the normalized collision energy of which was set as $30 \mathrm{eV}$.

\section{Data analysis}

All MS/MS data were analyzed using Maxquant1.3.0.5 implementing a Mascot search engine (Matrix Science, London, UK version 2.6.1). The mascot was set up to search the database P17429_fasciola_hepatica_33454_20170815.FASTA (total number of sequences: 33,454; download link: http://www. uniprot.org). The search parameters were set as follows: (i) enzyme: trypsin; (ii) missed cleavage sites: set to 2; (iii) a fixed modification: carbamidomethyl cysteine; and (iv) variable modifications: an oxidation of methionine (M), acetylation of protein N-term, phosphorylation of serine (S), threonine (T), and tyrosine $(\mathrm{Y})$. For proteins and peptides, the filter parameter false discovery rate (FDR) was set as $\leq 0.01$.

\section{Bioinformatic analysis}

BLAST analysis was performed using Blast2GO (http://www. blast2go.com), and the Gene Ontology (GO) functional categories were performed using the program InterProScan v5.15 (Conesa et al. 2005; Wang et al. 2019b). The InterProScan was utilized for identifying annotated genes features, including protein families, domains, functional sites, and GO terms from the InterPro database (Quevillon et al. 2005; Luo et al. 2021).

In addition, the Kyoto Encyclopedia of Genes and Genomes (KEGG) analysis (http://www.genome.jp/kegg/) were carried out to identify the enriched pathways. The classified pathways were shown in the hierarchical categories according to the data from the KEGG website.

\section{Results}

\section{Identification of phosphoproteins and phosphopeptides}

In this study, $\mathrm{TiO}_{2}$-dependent phosphopeptide enrichment method, LC-MS/MS analysis, and Mascot search were used, and a total of 1,030 phosphopeptides were identified, of which 1244 phosphosites represented 635 phosphoproteins of adult $F$. gigantica (Fig. 1A). The identified 1244 phosphosites consisted of 1086 serine phosphorylation (87.3\%), 145 threonine phosphorylation (11.7\%), and 13 tyrosine phosphorylation (1.0\%) (Fig. 1B, Supplementary Table 1). In particular, both hypothetical protein BN1106_s378B000167 and BN1106_s2053B000154 contained more than 10 serine phosphosites and with 6 threonine phosphosites and one tyrosine phosphosite, respectively. In addition, metabolic enzymes like glycogen synthase (BN1106_s2333B000155) and phosphatidylinositol-4-phosphate 3-kinase (BN1106_s403B000429) were rich in these phosphorylation modifications. The phosphoserine, phosphothreonine, or phosphotyrosine modification ratios found in this work were similar to those previously described in other organisms (Wang et al. 2020; Marchini et al. 2011; Hu et al. 2021) and most (90.0\%, 1120/1244) phosphosites located at the second amino acid of the peptides or after the position (Fig. 1A, Supplementary Table 1). Out of the identified phosphopeptides, 249 peptides $(24.2 \%)$ were found phosphorylated at $\geq 2$ phosphosites, and most peptides (82.5\%) contain $\geq 10$ amino acids (Fig. 1A, Supplementary Table 2). The phosphoproteins account for 3\% of F. gigantica proteins (Pandey et al. 2020). Therein, 196 (30.9\%) phosphoproteins contained $\geq 2$ phosphopeptides, while BN1106_s323B000258 (myosin heavy chain 6/7) and BN1106_s378B000167 (hypothetical protein) contained more than 10 phosphopeptides (Fig. 1A, Supplementary Table 3). Furthermore, a number $(n=25)$ of phosphorylated protein kinases that covered $5.5 \%$ of total F. gigantica kinases (Das et al. 2020) were identified including mitogen-activated protein kinase (MAPK) and calcium/calmodulin-dependent protein kinase (CAMK/CDPK) as well as serine/threonine/ tyrosine-protein kinase, etc.

\section{GO enrichment analysis}

Using the automatic annotation tool Blast2GO, functional annotations were conducted to characterize the identified phosphoproteins in this work. The GO enrichment analysis showed that these phosphoproteins were involved in a wide variety of biological processes (BP) including cellular, 
Fig. 1 Overall characteristics of the phosphoproteomics of $F$. gigantica. A General description about remarks of F. gigantica phosphoproteins, phosphopeptides, and phosphosites. B Distribution of identified phosphosites on serine, threonine, and tyrosine
Fig. 2 Gene ontology (GO) term distribution of $F$. gigantica phosphoproteins in three categories (biological process, molecular function, and cellular component). GO annotation and categorization were performed using Blast2GO
A

\begin{tabular}{|c|c|c|}
\hline $\begin{array}{c}\text { Categories } \\
\text { (Phosphorylation) }\end{array}$ & $\begin{array}{c}\text { Total } \\
\text { number }\end{array}$ & Remarks \\
\hline Proteins & 635 & $30.9 \% \geq 2$ peptides \\
\hline Peptides & 1030 & $24.2 \% \geq 2$ sites \\
\hline Sites & 1244 & $\begin{array}{c}90.0 \% \geq 2 \text { nd position } \\
\text { of peptide } \text {-terminal }\end{array}$ \\
\hline
\end{tabular}

B
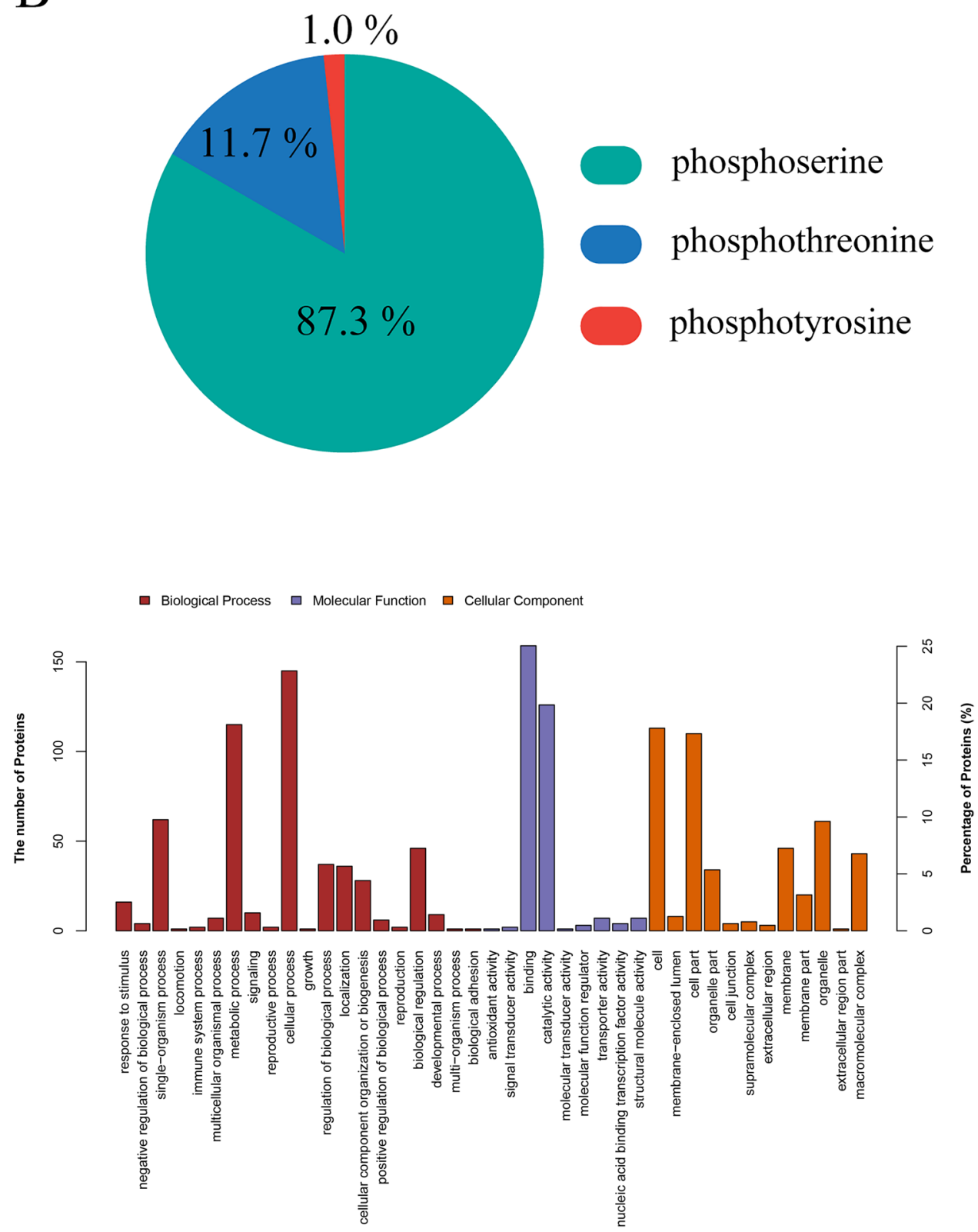
metabolic, or single-organism processes and biological regulation, localization, cellular component organization or biogenesis, as well as in response to stimulus (Fig. 2, Supplementary Table 4). Regarding the subcellular localization of the phosphoproteins, the GO annotations based on level 2 and level 5 inferred that these proteins were found predominantly in cellular components (CC) like membranes, organelles, and nuclei. Interestingly, only a small portion of them were predicted to be located in extracellular regions (Fig. 2, Supplementary Table 5), which made the finding reliable since extracellular components were not typically regulated by phosphorylation. According to the localization, the molecular functions (MF) of phosphoproteins was predominantly $(51.3 \%)$ associated with term "binding" based on level 2 , followed by different activities such as catalytic activity $(40.6 \%)$ and both transporter activity and structural molecule activity that occupied a small proportion (Fig. 2, Supplementary Table 6). In total, we found that half (317/635) of the phosphoproteins were annotated in at least one GO category (BP, CC, or MF), and one-third of them were annotated in at least two GO categories (Supplementary Table 7).

\section{KEGG pathway annotation}

In order to determine whether these phosphoproteins act independently in related signaling pathways or work in concert with other proteins, the KEGG database was used to annotate these phosphoproteins to help us understand their potential functions. The KEGG annotation inferred that the most enriched pathways of the phosphoproteins included tight junction, spliceosome, and RNA transport, and each one contained 15 identified proteins (Fig. 3, Supplementary Table 8). PI3K-akt signaling pathway, oxytocin signaling pathway, cAMP and cGMP-PKG signaling pathway, as well as hippo and insulin signaling pathway were ranked in the top 20 of KEGG pathway annotation. Additionally, parts of phosphoproteins were also predicted to participate in the focal adhesion and protein processing as well as carbon metabolism (Supplementary Table 8). Furthermore, we found that $30.4 \%(193 / 635)$ of phosphoproteins were annotated in at least one KEGG pathway and $16.9 \%$ was annotated in at least two KEGG pathways (Supplementary Table 8). Therein, metabolic enzymes, such as adenylate cyclase 9 (ADCY9) and hexokinase (HK), and protein kinases including p21-activated kinase 1 (PAK1), Rho-associated protein kinase 2 (ROCK2), mitogen-activated protein kinase 1/3 (MAPK1/3), and serine/threonine-protein kinase mTOR, as well as effectors like calmodulin $(\mathrm{CaM})$ and heat shock protein 90 (HSP90) were predicted to be annotated in more than 10 KEGG pathways (Supplementary Table 9).

\section{Discussion}

As a food-borne trematode, $F$. gigantica is responsible for the hepatobiliary disease fascioliasis (Good and Scherbak 2021). In terms of parasite growth and signal transduction, protein phosphorylation or dephosphorylation catalyzed by specialized protein kinases or phosphatases of $F$. gigantica played essential roles in corresponding cellular processes, which helps maintain the cell homeostasis of parasites (Das et al. 2020). Thus, to study the phosphoproteomics profile is of great significance for exploring the roles of these proteins
Fig. 3 Enrichment analysis of top 20 KEGG pathways of $F$. gigantica phosphoproteins

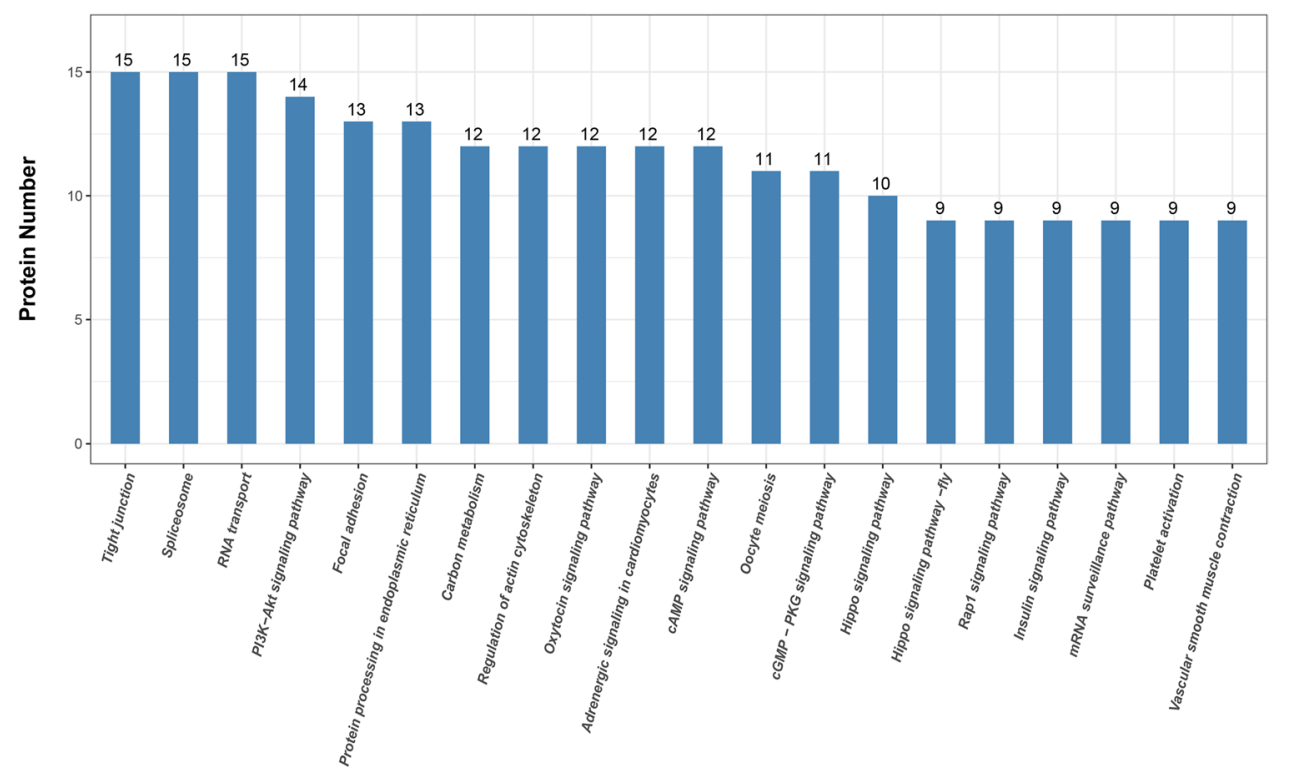

KEGG Pathways (Top 20) 
in the growth and development of parasite and for developing intervention strategies for fascioliasis.

In this work, a global phosphoproteomics analysis of $F$. gigantica was characterized, although it is probably not yet exhaustive. The majority of the detected phosphorylation events (>99\%) occurred on serine and threonine residues (Supplementary Table 1), and glycogen synthase was rich in serine and threonine phosphorylation in particular. Glycogen synthase was phosphorylated by a conserved glycogen synthase kinase 3 (GSK3) in eukaryotes, and GSK3 acted as a downstream component of the Wnt pathway during embryogenesis (Vital et al. 2010). A study demonstrated that GSK3 was involved in regulation of oogenesis and embryogenesis of Rhodnius prolixus (a blood-feeding insect as a media of Trypanosoma), and the activity and transcription levels of GSK3 were related to glycogen content (Mury et al. 2016). In Schistosoma japonicum, GSK3 $\beta$ (one GSK3 isoform) was highly expressed in the eggs (Liu et al. 2020a). GSK3 $\beta$ RNAi led to a significant decrease in kinase activity and cell viability, showing GSK3 $\beta$-dependent phosphorylation played an important role in worm survival (Liu et al. 2020a). In that case, future developmental phosphoproteomics analyses supplementing with knockdown experiments are needed to confirm whether the hyper-phosphorylated glycogen synthase correlates with the development and survival of $F$. gigantica or not in a certain stage. On the other hand, previous studies had found that the mutations in important phosphosites might result in the termination of the phosphorylation, leading to disorders of biological processes (Liu et al. 2020b). So an additional point mutation in glycogen synthase will be used to test the essentiality of the serine or threonine phosphorylation and further explore its functional domains.

For phosphoproteins identified in this work, cathepsin L1 (CL1) and glutathione S-transferase (GST) were regarded as lead vaccine candidates against liver flukes (McManus 2020), and in Fasciola spp., they could be efficiently inhibited by chalcones and two anti-parasitic agents (thymoquinone and curcumin), respectively (Ferraro et al. 2016; Ullah et al. 2017). Given that CL1 and GST showed good effects on the prevention and treatment of fascioliasis, the use of multistage-specific cocktails with better antigen-delivery systems and more small-molecule chemicals to elicit lethal or sub-lethal phenotypes would be considerable merit in vaccine development and drug target predictions in the future. Moreover, the phosphorylated effectors such as 14-3-3 and thioredoxin were also identified, and previous studies found that they could be recognized by sera from goats or mice experimentally infected with $F$. gigantica (Tian et al. 2018; Changklungmoa et al. 2020), making them considerable for immunodiagnostic candidates for fascioliasis.

According to KEGG annotation, nearly 7\% of phosphoproteins indicating multiple roles in different biological processes were predicted to be annotated in more than 5 KEGG pathways (Supplementary Table 9). Therein, malate dehydrogenase (MDH) of $F$. gigantica was a single cytosolic enzyme to catalyze the reversible oxidation of malate to oxaloacetate using $\mathrm{NAD}^{+}$(Chetri et al. 2020). Been as an isoenzyme, the pattern of FgMDH was the same as FhMDH due to similar relative mobilities (Sarkari et al. 2016). The superimposition structure model of FgMDH and human MDH showed overall structural similarity in the active site loop region, while the conformation of the residues was different (Chetri et al. 2020). Combining the structure model and our annotations in FgMDH in metabolic pathways such as pyruvate metabolism, cysteine and methionine metabolism, and glyoxylate and dicarboxylate metabolism, the roles of FgMDH can be used to better understand the biochemistry of $F$. gigantica. P21-activated protein kinase (PAKs) had long been established to play important roles in vital cellular functions such as proliferation, survival, and motility (Arias-Romero and Chernoff 2008). Emerging evidence showed host PAK1 increased cell survival during the stage of virus infection (Van den Broeke et al. 2010), and recent study found PAK1 enhanced macrophage activation, resulting in promoting of Th17 cell response during Schistosoma japonicum infection (Chang et al. 2020), both of which guide the future research on the immunomodulation roles of host PAK1 and parasitic PAK1 in Fasciola infection. To further understand the functions of $F$. gigantica phosphoproteins annotated in more than $10 \mathrm{KEGG}$ pathways, we took them in comparison with protozoa and helminths to identify possible connections between these phosphoproteins and biological processes of the parasites. Based on recent reports (Table 1), calcium/calmodulin-dependent protein kinase (CAMK4/ CDPK4) and 14-3-3 protein of Plasmodium falciparum participated in controlling the parasite invasion and regulating the assembly of signaling complexes, respectively (Fang et al. 2018; More et al. 2020). In Leishmania, mitogen-activated protein kinase 1 (MAPK1) and heat shock protein 90 (HSP90) played important roles in stress regulation and nascent protein synthesis (Kaur et al. 2021; Kalesh et al. 2021). Additionally, the metabolic enzymes acted as sensors to regulate the metabolites and metabolic flux, contributing to parasite growth (Liang et al. 2020; Rodriguez-Saavedra et al. 2021). Camodulins (CaMs) were involved in fundamental processes including the phosphorylation of protein kinases, gene transcription, and calcium transport (Hoeflich and Ikura 2002). In Schistosoma mansoni, calmodulin had been implicated in egg hatching, miracidial transformation, and larval development (Guidi et al. 2015). While in F. hepatica, the FhCaMs functioned as $\mathrm{Ca}^{2+}$ modulators were proved to be important for the growth and movement of juvenile fluke (McCammick et al. 2016). Recent research clarified calmodulin of Caenorhabditis elegans was implicated in the plasticity impairment of high-activity neurons with age, 
Table 1 Recent researches on the functions of phosphoproteins in other protozoa and helminths

\begin{tabular}{|c|c|c|c|c|}
\hline \multicolumn{2}{|l|}{ Phosphoproteins } & \multirow{2}{*}{$\begin{array}{l}\text { Functions } \\
\text { A glucose sensor to regulate the gly- } \\
\text { colysis flux and ATP production }\end{array}$} & \multirow{2}{*}{$\begin{array}{l}\text { Parasites } \\
\text { Trypanosoma brucei }\end{array}$} & \multirow{2}{*}{$\begin{array}{l}\text { References } \\
\text { Rodriguez-Saave- } \\
\text { dra et al. } 2021\end{array}$} \\
\hline Metabolic enzymes & Hexokinase (HK) & & & \\
\hline & Pyruvate dehydrogenase (PDH E1 $\alpha$ ) & $\begin{array}{l}\text { Contributes to parasite growth via } \\
\text { enhanced fatty acid synthesis }\end{array}$ & Toxoplasma gondii & Liang et al. 2020 \\
\hline \multirow[t]{2}{*}{ Protein kinases } & $\begin{array}{l}\text { Calcium/calmodulin-dependent pro- } \\
\text { tein kinase } 4 \text { (CAMK4/CDPK4) }\end{array}$ & $\begin{array}{l}\text { Controls parasite motility and host } \\
\text { cell invasion }\end{array}$ & Plasmodium falciparum & Fang et al. 2018 \\
\hline & $\begin{array}{l}\text { Mitogen-activated protein kinase } 1 \\
\text { (MAPK1) }\end{array}$ & $\begin{array}{l}\text { Acts in regulation of stress machin- } \\
\text { ery through post-translational } \\
\text { modifications }\end{array}$ & Leishmania donovani & Kaur et al. 2021 \\
\hline \multirow[t]{3}{*}{ Effectors } & Calmodulin & $\begin{array}{l}\text { Declines the plasticity of high-activ- } \\
\text { ity neurons }\end{array}$ & Caenorhabditis elegans & Li et al. 2020 \\
\hline & $14-3-3$ & $\begin{array}{l}\text { Binds phosphorylated PKAr and } \\
\text { CDPK1 to mediate the assembly of } \\
\text { signaling complexes }\end{array}$ & Plasmodium falciparum & More et al. 2020 \\
\hline & Heat shock protein 90 (HSP90) & Regulates nascent protein synthesis & Leishmania mexicana & Kalesh et al. 2021 \\
\hline
\end{tabular}

indicating a different but novel role in neuronal activity $(\mathrm{Li}$ et al. 2020). In short, the relationship between these proteins of different parasites will help us better understand the phosphoproteomics profile of $F$. gigantica.

\section{Conclusions}

In this work, we defined the global phosphoproteomics of a tropical liver fluke by a phosphorylation-specific enrichment technique. From the mass spectrometry and data analysis, we identified several phosphoproteins and predicted their functions, which enhanced our knowledge of key biological processes in $F$. gigantica. With a view towards future exploration into the phosphoproteomics analyses of this parasite at different life-cycle stages, new effective intervention strategies will be developed for the control of fascioliasis.

Supplementary Information The online version contains supplementary material available at https://doi.org/10.1007/s00436-021-07422-2.

Acknowledgements We would like to thank the Shanghai Applied Protein Technology Co. Ltd. (Shanghai, China) for performing the mass spectrometry analysis.

Author contribution SYH and MP conceived and designed the study. MP, SYB, JZG, and DDL performed the laboratory analyses. QWJ and JPT analyzed the data. All authors critically appraised and interpreted the results. MP drafted the first version of the manuscript. All authors provided feedback on the manuscript and read and approved the final version.

Funding This work was supported by the National Key Research and Development Program of China (Grant No. 2017YFD0501200) and a project funded by the Priority Academic Program Development of Jiangsu Higher Education Institutions (Veterinary Medicine); the funders were not involved in the study design; collection, analysis, and interpretation of the data; or in writing the manuscript.

\section{Declarations}

Ethics approval and consent to participate The experiments were performed in strict accordance with the recommendations of the Guide for the Care and Use of Laboratory Animals of the Ministry of Science and Technology of China. All experimental animal procedures and protocols (Approval No. 14567) were approved by the Institutional Animal Care and Use Committee of Yangzhou University.

Competing interests The authors declare no competing interests.

\section{References}

Arias-Romero LE, Chernoff J (2008) A tale of two Paks. Biol Cell 100(2):97-108. https://doi.org/10.1042/BC20070109

Broncel M, Treeck M (2020) Label-based mass spectrometry approaches for robust quantification of the phosphoproteome and total proteome in Toxoplasma gondii. Methods Mol Biol 2071:453-468. https://doi.org/10.1007/978-1-4939-9857-9_23

Calvani NED, Slapeta J (2021) Fasciola species introgression: just a fluke or something more? Trends Parasitol 37(1):25-34. https:// doi.org/10.1016/j.pt.2020.09.008

Chang $\mathrm{H}$ et al (2020) P21 activated kinase-1 (PAK1) in macrophages is required for promotion of Th17 cell response during helminth infection. J Cell Mol Med 24(24):14325-14338. https://doi.org/ $10.1111 /$ jcmm. 16050

Changklungmoa $\mathrm{N}$ et al (2020) A novel Thioredoxin-related protein 14 from Fasciola gigantica has an immunodiagnostic potential for fasciolosis. Acta Trop 207:105471. https://doi.org/10.1016/j. actatropica.2020.105471

Chetri PB, Shukla R, Tripathi T (2020) Identification and characterization of cytosolic malate dehydrogenase from the liver fluke Fasciola gigantica. Sci Rep 10(1):13372. https://doi.org/10. 1038/s41598-020-70202-y

Conesa A, Gotz S, Garcia-Gomez JM, Terol J, Talon M, Robles M (2005) Blast2GO: a universal tool for annotation, visualization and analysis in functional genomics research. Bioinformatics 21(18):3674-3676. https://doi.org/10.1093/bioinformatics/ bti610 
Cwiklinski K, O’Neill SM, Donnelly S, Dalton JP (2016) A prospective view of animal and human Fasciolosis. Parasite Immunol 38(9):558-568. https://doi.org/10.1111/pim.12343

Das KC, Kalita P, Tripathi T (2020) Genome-wide identification and characterization of eukaryotic protein kinases. Front Biosci (landmark Ed) 25:1787-1827

Day EK, Sosale NG, Lazzara MJ (2016) Cell signaling regulation by protein phosphorylation: a multivariate, heterogeneous, and context-dependent process. Curr Opin Biotechnol 40:185-192. https://doi.org/10.1016/j.copbio.2016.06.005

Efstathiou A, Smirlis D (2021) Leishmania protein kinases: important regulators of the parasite life cycle and molecular targets for treating leishmaniasis. Microorganisms 9(4). https://doi.org/10.3390/ microorganisms 9040691

Fang $\mathrm{H}$ et al (2018) Epistasis studies reveal redundancy among calcium-dependent protein kinases in motility and invasion of malaria parasites. Nat Commun 9(1):4248. https://doi.org/10. 1038/s41467-018-06733-w

Ferraro F et al (2016) Identification of chalcones as Fasciola hepatica cathepsin L inhibitors using a comprehensive experimental and computational approach. PLoS Negl Trop Dis 10(7):e0004834. https://doi.org/10.1371/journal.pntd.0004834

Gaji RY, Sharp AK, Brown AM (2021) Protein kinases in Toxoplasma gondii. Int J Parasitol 51(6):415-429. https://doi.org/10.1016/j. ijpara.2020.11.006

Good R, Scherbak D (2021) Fascioliasis StatPearls. Treasure Island (FL)

Guidi A et al (2015) Application of RNAi to genomic drug target validation in schistosomes. PLoS Negl Trop Dis 9(5):e0003801. https://doi.org/10.1371/journal.pntd.0003801

Hayward AD, Skuce PJ, McNeilly TN (2021) The influence of liver fluke infection on production in sheep and cattle: a meta-analysis. Int J Parasitol https://doi.org/10.1016/j.ijpara.2021.02.006

Hirst NL, Nebel JC, Lawton SP, Walker AJ (2020) Deep phosphoproteome analysis of Schistosoma mansoni leads development of a kinomic array that highlights sex-biased differences in adult worm protein phosphorylation. PLoS Negl Trop Dis 14(3):e0008115. https://doi.org/10.1371/journal.pntd.0008115

Hoeflich KP, Ikura M (2002) Calmodulin in action: diversity in target recognition and activation mechanisms. Cell 108(6):739-742. https://doi.org/10.1016/s0092-8674(02)00682-7

$\mathrm{Hu} \mathrm{Y}$ et al (2021) Quantitative proteomics and phosphoproteomic analyses of mouse livers after tick-borne Babesia microti infection. Int J Parasitol 51(2-3):167-182. https://doi.org/10.1016/j. ijpara.2020.09.002

Humphrey SJ, James DE, Mann M (2015) Protein phosphorylation: a major switch mechanism for metabolic regulation. Trends Endocrinol Metab 26(12):676-687. https://doi.org/10.1016/j.tem.2015. 09.013

Kalesh K, Sundriyal S, Perera H, Cobb SL, Denny PW (2021) Quantitative proteomics reveals that $\mathrm{Hsp} 90$ inhibition dynamically regulates global protein synthesis in Leishmania mexicana. mSystems 6(3). https://doi.org/10.1128/mSystems.00089-21

Kaur P, Anand A, Bhat A, Maras JS, Goyal N (2021) Comparative phosphoproteomic analysis unravels MAPK1 regulated phosphoproteins in Leishmania donovani. J Proteomics 240:104189. https://doi.org/10.1016/j.jprot.2021.104189

Larsen MR, Jensen SS, Jakobsen LA, Heegaard NH (2007) Exploring the sialiome using titanium dioxide chromatography and mass spectrometry. Mol Cell Proteomics 6(10):1778-1787. https://doi. org/10.1074/mcp.M700086-MCP200

Li Q, et al. (2020) High neural activity accelerates the decline of cognitive plasticity with age in Caenorhabditis elegans. Elife 9. https:// doi.org/10.7554/eLife.59711

Liang X et al (2020) Acquisition of exogenous fatty acids renders apicoplast-based biosynthesis dispensable in tachyzoites of
Toxoplasma. J Biol Chem 295(22):7743-7752. https://doi.org/ 10.1074/jbc.RA120.013004

Liu J et al (2020a) Identification of Schistosoma japonicum GSK3 beta interacting partners by yeast two-hybrid screening and its role in parasite survival. Parasitol Res 119(7):2217-2226. https://doi.org/10.1007/s00436-020-06731-2

Liu W et al (2020b) Protein phosphorylation networks in spargana of Spirometra erinaceieuropaei revealed by phosphoproteomic analysis. Parasit Vectors 13(1):248. https://doi.org/10.1186/ s13071-020-04119-w

Luo X et al (2021) High-quality reference genome of Fasciola gigantica: insights into the genomic signatures of transposon-mediated evolution and specific parasitic adaption in tropical regions. PLoS Neg1 Trop Dis 15(10):e0009750. https://doi.org/10.1371/ journal.pntd.0009750

Mann M, Jensen ON (2003) Proteomic analysis of post-translational modifications. Nat Biotechnol 21(3):255-261. https://doi.org/ 10.1038/nbt0303-255

Marchini FK et al (2011) Profiling the Trypanosoma cruzi phosphoproteome. PLoS ONE 6(9):e25381. https://doi.org/10.1371/ journal.pone.0025381

Marcos L, Maco V, Terashima A (2021) Triclabendazole for the treatment of human fascioliasis and the threat of treatment failures. Expert Rev Anti Infect Ther 19(7):817-823. https://doi. org/10.1080/14787210.2021.1858798

Mas-Coma S, Valero MA, Bargues MD (2019) Fascioliasis. Adv Exp Med Biol 1154:71-103. https://doi.org/10.1007/ 978-3-030-18616-6 4

McCammick EM et al (2016) Calmodulin disruption impacts growth and motility in juvenile liver fluke. Parasit Vectors 9:46. https:// doi.org/10.1186/s13071-016-1324-9

McManus DP (2020) Recent progress in the development of liver fluke and blood fluke vaccines. Vaccines (Basel) 8(3). https:// doi.org/10.3390/vaccines 8030553

More KR, et al. (2020) Phosphorylation-dependent assembly of a 14-3-3 mediated signaling complex during red blood cell invasion by Plasmodium falciparum merozoites. mBio 11(4). https:// doi.org/10.1128/mBio.01287-20

Mury FB et al (2016) Glycogen synthase kinase-3 is involved in glycogen metabolism control and embryogenesis of Rhodnius prolixus. Parasitology 143(12):1569-1579. https://doi.org/10. $1017 / \mathrm{S} 0031182016001487$

Pandey T et al (2020) Draft genome of the liver fluke Fasciola gigantica. ACS Omega 5(19):11084-11091. https://doi.org/10.1021/ acsomega.0c00980

Quevillon E, et al. (2005) InterProScan: protein domains identifier. Nucleic Acids Res 33(Web Server issue):W116-20. https://doi. org/10.1093/nar/gki442

Ren L, Li C, Shao W, Lin W, He F, Jiang Y (2018) TiO2 with tandem fractionation (TAFT): an approach for rapid, deep, reproducible, and high-throughput phosphoproteome analysis. J Proteome Res 17(1):710-721. https://doi.org/10.1021/acs.jproteome.7b00520

Rodriguez-Saavedra C, Morgado-Martinez LE, Burgos-Palacios A, King-Diaz B, Lopez-Coria M, Sanchez-Nieto S (2021) Moonlighting proteins: the case of the hexokinases. Front Mol Biosci 8:701975. https://doi.org/10.3389/fmolb.2021.701975

Sarkari B, Sedaghat B, Hatam GR (2016) Comparative study on isoenzyme patterns of Fasciola hepatica and Fasciola gigantica. Trop Biomed 33(3):462-468

Thingholm TE, Jorgensen TJ, Jensen ON, Larsen MR (2006) Highly selective enrichment of phosphorylated peptides using titanium dioxide. Nat Protoc 1(4):1929-1935. https://doi.org/10.1038/ nprot.2006.185

Tian AL et al (2018) A recombinant Fasciola gigantica 14-3-3 epsilon protein $(\mathrm{rFg} 14-3-3 \mathrm{e})$ modulates various functions of goat 
peripheral blood mononuclear cells. Parasit Vectors 11(1):152. https://doi.org/10.1186/s13071-018-2745-4

Ullah R et al (2017) Anthelmintic potential of thymoquinone and curcumin on Fasciola gigantica. PLoS ONE 12(2):e0171267. https:// doi.org/10.1371/journal.pone.0171267

Van den Broeke C, Radu M, Chernoff J, Favoreel HW (2010) An emerging role for $\mathrm{p} 21$-activated kinases (Paks) in viral infections. Trends Cell Biol 20(3):160-169. https://doi.org/10.1016/j. tcb.2009.12.005

Vital W et al (2010) Germ band retraction as a landmark in glucose metabolism during Aedes aegypti embryogenesis. BMC Dev Biol 10:25. https://doi.org/10.1186/1471-213X-10-25

Wang T et al (2019a) High throughput LC-MS/MS-based proteomic analysis of excretory-secretory products from short-term in vitro culture of Haemonchus contortus. J Proteomics 204:103375. https://doi.org/10.1016/j.jprot.2019.05.003

Wang ZX et al (2019b) iTRAQ-based global phosphoproteomics reveals novel molecular differences between toxoplasma gondii strains of different genotypes. Front Cell Infect Microbiol 9:307. https://doi.org/10.3389/fcimb.2019.00307

Wang T et al (2020) The developmental phosphoproteome of Haemonchus contortus. J Proteomics 213:103615. https://doi.org/10. 1016/j.jprot.2019.103615

Wu K, Zhai X, Huang S, Jiang L, Yu Z, Huang J (2021) Protein kinases: potential drug targets against Schistosoma japonicum. Front Cell Infect Microbiol 11:691757. https://doi.org/10.3389/ fcimb.2021.691757

Publisher's note Springer Nature remains neutral with regard to jurisdictional claims in published maps and institutional affiliations. 